\title{
Outcomes of a pregnancy after fifteen cycles of treatment with IVF and ET
}

\author{
Astrit Malush Gashi \\ University Clinical Center of Kosovo, Obstetrician and Gynecological Clinic, Pristine, Kosovo
}

\begin{abstract}
In vitro fertilization and embryo transfer is the traditional method of infertility treatment, consisting of in vitro fertilization (IVF) and embryo transfer (ET) in the uterine cavity. Infertility is defined as the absence of pregnancy after regular unprotected intercourse throughout one year. The main causes of infertility are: ovulatory dysfunction, age and diminished ovarian reserve, male factors, tubal factors, uterine factors, cervical factors, immunological factors, systemic diseases (chronic and autoimmune diseases) and idiopathic factors. In our case, we are dealing with ovulatory dysfunction (PCOS). We present the case of a 32 year-old Kosovo with a twelveyear history of infertility, who was treated with fifteen cycles of long protocols of in vitro fertilization and embryo transfer and in spite of multiple issues throughout pregnancy, finally lead to a successful parturition. The patient was hospitalized in the Clinic of Obstetrics and Gynecology at Pathology Department for acute abdominal pain at 26 weeks of gestation after undergoing the in vitro fertilization and embryo-transfer procedure. The reference diagnosis was: olygohydramnios, pregnancy-induced hypertension, twelve-year history of primary infertility, history of fifteen IVF and ET.
\end{abstract}

Keywords: Infertility, ovulatory dysfunction, vitro fertilization, embryo transfer

\section{Introduction}

According to ASRM and ESHRE consensus, primary infertility is defined as "failure to achieve a successful pregnancy after 12 months or more of appropriate, timed unprotected intercourse in women without any pregnancy before" while "Recurrent pregnancy loss is a disease distinct from infertility, defined by two or more failed pregnancies" [1]. Infertility can be primary and secondary. Primary infertility is when a woman is unable to ever bear a child, either due to the inability to become pregnant or the inability to carry a

Received: March 2016; Accepted after review: June 2016; Published: June 2016.

*Corresponding author: Astrit Malush Gashi MD, University Clinical Center of Kosovo, Obstetrician and Gynecological Clinic Pristine, 13.K. e Diellit St. Afrim Zhitia BLL 3 Hy 1, No.11-1, Pristine 10000, Kosovo. Email: astritgashi772@gmail.com pregnancy leading to a successful parturition. Thus, women whose pregnancy spontaneously miscarries or whose pregnancy results in a still born child, without ever having had a live birth would present with primarily infertility [2]. A woman who is unable to bear a child, either due to the inability to become pregnant or the inability to carry a pregnancy to a live birth, following either a previous pregnancy or a previous ability to carry a pregnancy to a live births is classified as having secondary infertility. Thus, those who repeatedly, spontaneously miscarry or whose pregnancy results in a stillbirth, or following a previous pregnancy or a previous ability to do so, are then not unable to carry a pregnancy to a live birth would be secondarily infertile [2]. Clinical definitions are designed for early detection and treatment of infertility [3, 4]. In vitro fertilization and embryo transfer is the traditional method of infertility treatment. In 
vitro fertilization with or without intracytoplasmic sperm injection (ICSI) is the most successful treatment for infertility. The main causes of infertility are: ovulatory dysfunction, age and diminished ovarian reserve, male factors, tubal factors, uterine factors, cervical factors, immunological factors, systemic diseases (chronic and autoimmune diseases) and idiopathic factors. In our case, we are dealing with ovulatory dysfunction (PCOS) and damaged fallopian tubes. Infertility can lead to distress and depression, as well as discrimination and ostracism [5, 6].

\section{Case report}

A 32-year-old female was admitted to the Pathology Department of our hospital with acute abdominal pain at 26 weeks of gestation after undergoing the IVF and ET procedure. The reference diagnosis was: oligohydramnios, pregnancy-induced hypertension, twelve-year history of primary infertility, history of fifteen IVF and ET (cycles of long protocols). She was a non-smoker, had a negative history of alcohol or drug consumption, with no history of abdominal surgery. She had AB positive blood group. Body mass index: 31.8. The patient had a stable life partner for twelve years; she has no history of spontaneous pregnancy, but regular visits to the gynecologist after a year of infertility, being diagnosed with polycystic ovarian syndrome. The diagnosis was based on a combination of clinical, ultrasound and biochemical criteria. She had irregular menses in the form of oligomenorrhea, obesity, acne, hirsutism. Ultrasound evaluation revealed thirteen ring-shaped follicles with diameter of about 2-7 mm, mainly located in the periphery, along with stromal hyperplasia and an increase in the ovarian volume. Luteinizing hormone $(\mathrm{LH})>12 \mathrm{UI} / \mathrm{L}$ and testosterone levels were increased, while dehydroepiandrosterone sulfate (DHEAS) was slightly increased. Her final diagnosis was polycystic ovarian syndrome (PCOS). She also had a history of three courses of antibiotherapy (with azithromycin twice, and once with doxycycline 7 day) following Chlamydia trachomatis infection. The diagnostic laparoscopy showed damage of both fallopian tubes. She followed all forms of treatment of polycystic ovarian syndrome, without therapeutic success. Following several abortions, the couple was genetically tested, but kariotyping resulted normal. She was also assessed for uterine abnormalities, initially through hysterosalpingography procedure, then with hysteroscopy, without identifying any uterine cavity malformation. During the twelve years of infertility, she followed fifteen courses of IVD and ET (cycles of long protocols), which resulted in four pregnancies, among which three resulted in spontaneous abortions (less than six weeks of pregnancy), and the latest pregnancy was successful. All IVF cycles were performed according to routine IVF procedures. Ovulation induction was achieved with Metrodin HP (150-225 IU), following a long protocol of pituitary desensitization with Buserelin acetate (stimulation lasted 8 days, from third day until the tenth day of menstrual cycle). Human chorionic gonadotrophin (HCG) Pregynl was administered when there were at least four follicles with mean diameter of 17 $\mathrm{mm}, 36$ hours before egg retrieval. Mature, metaphase 2 oocytes obtained by vaginal ultrasonographic-guided aspiration were cultured in universal IVF medium, at $37^{\circ} \mathrm{C}$, and in $5 \% \mathrm{CO} 2$ integral. Fertilization was recorded after 12-16 hours if two pronuclei were detected. Embryo transfer procedure was done on day twenty-one. With IVF and ET procedure, one embryo is placed in the uterine cavity. Embryo-transfer procedure lasted 5 day, and embryo it was placed in the uterine cavity in the blastocyst stage. If the selected embryo is healthy, highly-developed, and strong, there is a much greater chance of successful implantation and resulting in a successful pregnancy. Fertilization rate was 51, 2\%. After IVF and ET procedures, she made systematic visits every 4 weeks, with no issues until 26 weeks gestational age, when the patient was admitted to our hospital for acute abdominal pain. She described her pain as originating in the lower abdomen, of severe, sharp and constant intensity, without association with nausea, emesis, dyspnea or changes with the patient's body position. The 
initial physical examination revealed a blood pressure of $150 / 95 \mathrm{mmHg}$, a heart rate of 77 beats per minute and a temperature of 37.1 ${ }^{\circ} \mathrm{C}$. Upon palpation, the patient had slight abdominal tenderness. The gynecological examination revealed: modified uterine tonus, with contractions at uterine wall, normal length, closed cervix. Fetal biometric corresponded to $25+1$ weeks of gestation, the placenta on anterior wall, with no pathological features. The amniotic fluid Index was $8,7 \mathrm{~cm}$ and Doppler examination of the umbilical artery was within normal values. Routine laboratory tests: complete blood count, urine biochemical analysis, urine culture, vaginal smear, biochemistry, CRP, hemostasis tests resulted in normal values. During 8 weeks of monitoring, clinical examination, ultrasonography, laboratory tests and additional required examinations were within normal range. The patient was administered antihypertensive (Methyldopa 250mg tablets, three times a day), anti-coagulants (Fraxiparine $2850 \mathrm{Ul} / 0.3 \mathrm{ml}$ sol. injection for prophylaxis treatment), corticoid (Dexamethasone $6 \mathrm{mg}$ injections, two doses per day, for two days), tocolytic (Indomethacin suppositories $100 \mathrm{mg}$, for three days) and hormonal therapy (Utrogestan $100 \mathrm{mg}$, tablets) and vitamins. At week $34+3$ pregnancy, due

\section{References}

1. Gianaroli L, Racowsky C, Geraedts $\mathrm{J}$ et al. Best practices of ASRM and ESHRE: a journey through reproductive medicine. Fertil Steril 2012; 98(6):1380-1394.

2. Mascarenhas $M N$, Flaxman $S R$, Boerma $T$, et al. National, regional, and global trends in infertility prevalence since 1990: a systematic analysis of 277 health surveys. PLoS Med 2012; 9(12):e1001356.

3. Zegers-Hochschild F, Adamson GD, de Mouzon J, et al. The International Committee for Monitoring Assisted Reproductive Technology (ICMART) and the World Health Organization (WHO) revised glossary on ART to Fetal Non-Stress test identifying reduced amniotic fluid, after adequate information, with the approval of the patient, a Caesarean delivery was decided; the patient underwent spinal anesthesia, giving birth to a child weighing 2500 grams, $49 \mathrm{~cm}$ length and Apgar score: $7 / 8$. The patient and her new-born were discharged after 3-day hospitalization, due to positive outcome.

\section{Discussion and conclusion}

The number of infertile couples has increased worldwide, due to population growth. In 2010, an estimated 48.5 million couples worldwide were infertile. IVF followed by $E T$ is the traditional method of infertility treatment. IVF with or without intracytoplasmic sperm injection (ICSI) is the most successful treatment for infertility. IVF and ET are used for treating infertility in patients, who have damaged fallopian tubes, male factor infertility, endometriosis, or unexplained infertility. In the presented case, we were dealing with ovulatory dysfunction (PCOS) and damaged fallopian tubes. The purpose of this case report is to prove that, after twelve years of infertility and fifteen laborious procedures of IVF and ET to treat infertility, it is possible to finally lead to a successful pregnancy.

terminology, 2009. Hum Reprod 2009; 24:2683-2687.

4. Rowe $\mathrm{PJ}$, Comhaire $\mathrm{FH}$, Hargreave TB, Mellows HJ. WHO manual for the standardized investigation and diagnosis of the infertile couple. Cambridge: Cambridge University Press, 1993.

5. Cui W. Mother or nothing: the agony of infertility. Bull World Health Organ 2010 88(12):881-882.

6. Chachamovich JR, Chachamovich E, Ezer H, et al. Investigating quality of life and healthrelated quality of life in infertility: a systematic review. J Psychosom Obstet Gynaecol 2010; 31:101-110. 